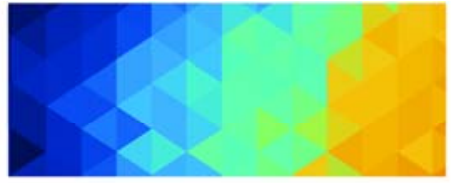

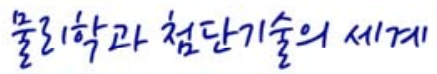

\title{
Large Matter-Antimatter Differences in B-meson Decays
}

DOI: $10.3938 /$ PhiT. 25.012

Large matter-antimatter differences (i.e., $C P$ violations) in the Laws of Nature are necessary to account for the matter-dominated present-day Universe. We, and, as far as we can tell, all discernible material in the Universe are comprised of matter; the minute amounts of antimatter that are seen in nature have, at best, a transitory existence. Although a tiny violation of $C P$ was discovered in the decays of neutral K-mesons in 1964, mechanisms to incorporate $C P$ violation into the Standard Model for particle physics remained elusive until 1973, when Kobayashi and Makawa proposed that this could be accomplished by introducing an irreducible complex phase into the quark-flavor mixing matrix. This could only be accomplished if the number of quark flavors was six, twice as many as the three quark flavors that were known at the time. Subsequently, six quark flavors were established and the Kobayashi-Maskawa mechanism was generally considered to be the most natural way to accommodate $C P$ violation into the theory. The Belle experiment was specifically designed to test the Kobayashi-Maskawa idea by looking for large $C P$-violating asymmetries in certain $B$ meson decay modes. Results reported by Belle (and the BaBar experiment) in Summer 2001 verified Kobayashi and Maskawa's proposal and led to their Nobel prizes in 2008. In this report I review some background on $C P$ violation, describe the Kobayashi Maskawa mechanism for $C P$ violation, and explain how it was experimentally verified.

\section{BACKGROUND}

The discrete operations of Parity ( $P$ : left $\leftrightarrow$ right), ChargeConjugation ( $C$ : particle $\leftrightarrow$ antiparticle) and Time-Reversal ( $T$ :

\section{저자약력}

Stephen Lars Olsen 교수는 University of Wisconsin에서 물리학으로 박 사위를 받았다.(1970) University of Rochester(1972-1992), University of Hawaii(1992-2009)를 거쳐 2009년부터 서울대학교 물리학과 교수 (2009-2014)로 재직하였으며 현재 기초과학연구원 지하실험연구단 명예연 구위원(2015-현재)으로 연구활동 중이다. 입자실험물리학 분야에서 탁월한 업 적을 남긴 연구자에게 수여되는 '2016 파노프스키 상을 수상하였다.

\section{Stephen Lars Olsen}

forward in time $\leftrightarrow$ backward in time) play major roles in particle physics. Prior to 1956, it was generally believed that the Laws of Nature had to be symmetric under each one of these transformations. However, puzzles in the decays of $K$ mesons led T. D. Lee and C. N. Yang to propose that Parity was violated by the Weak Interactions, ${ }^{[1]}$ a revolutionary proposal at that time, but one that was quickly confirmed by an experiment that discovered large Parity-violating asymmetries in radioactive decays of polarized ${ }^{60} \mathrm{Co}$ nuclei. ${ }^{[2]}$ Simultaneous studies of decays of muon particles found similarly large Parity-violating asymmetries that had opposite signs for muons $\left(\mu^{-}\right)$and antimuons $\left(\mu^{+}\right) \cdot{ }^{[3]}$ The opposite asymmetries for $\mu^{+}$and $\mu^{-}$indicated that Charge-Conjugation symmetry was also violated, but in such a way that the combined operation $C P$ seemed to remain a valid symmetry of nature. These results led to physics Nobel Prizes for Lee and Yang in 1957 and subsequent theoretical studies of, and experimental searches for, possible violations of $C P$.

If nature is symmetric under the operation of the triple product $C P T$, particle and antiparticle masses and lifetimes must be equal. In 1958, Okubo pointed out that if CPT is a good symmetry of nature but $C P$ is violated, the partial decay widths of a particle to particular final states could be different from those of its antiparticle; e.g., in the specific process discussed by Okubo: $\Gamma\left(\Sigma^{+} \rightarrow N \pi\right) \neq \Gamma\left(\bar{\Sigma}^{+} \rightarrow \bar{N} \bar{\pi}\right)$, while $\Gamma\left(\Sigma^{+} \rightarrow\right.$ all $)$ $=\Gamma\left(\bar{\Sigma}^{+} \rightarrow \overline{a l l}\right) \cdot{ }^{[4]}$ This was recognized by Sakharov in his classic 1967 paper $^{[5]}$ as an essential ingredient for explaining how a symmetric matter-antimatter condition that prevailed

\section{REFERENCES}

[1] T. D. Lee and C. N. Yang, Phys. Rev. 104, 254 (1956).

[2] C. S. Wu, E. Ambler, R. W. Hayward and R. P. Hudson, Phys. Rev. 105, 1413 (1957).

[3] R. L. Garwin, L. M. Lederman and M. Weinrich, Phys. Rev. 105, 1415 (1957).

[4] S. Okubo, Phys. Rev. 109, 984 (1958).

[5] A. D. Sakharov, Zh. EK. Teor. Fiz. 5, 32 (1967) (English translation: JETP Lett. 5, 24 (1967)). 

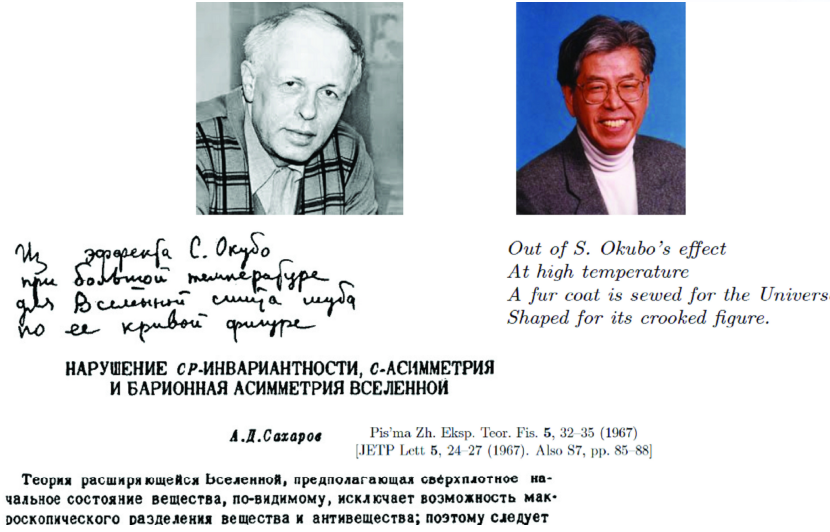

Fig. 1. When Sakharov completed his famous 1967 paper on "Violation of $C P$ invariance, $C$ asymmetry, and baryon asymmetry of the Universe," he gave his colleague Lev Okun a preprint with a small poem handwritten on it that identifies CPV as "S. Okubo's effect." This refers to a 1958 paper by Okubo that first pointed out that while $C P T$ invariance requires particle and antiparticle lifetimes to be equal, $C P$ violations would allow partial lifetimes to be different. ${ }^{[4]}$

right after the Big Bang could evolve into the decidedly asymmetric, matter-dominated universe that we live in today (see Fig. 1).

In a 1964 experiment at Brookhaven National Laboratory, a small $C P$ violation was discovered in the decays of neutral $K$ mesons, as evidenced by a small, but non-zero, decay rate of $K_{L}$ mesons (the "Long-lived" neutral $K$ meson) to $\pi^{+} \pi^{-}$; about twice per $10^{3}$ decays. This demonstrated that $C P$, i.e., matter-antimatter symmetry, is violated, probably by the weak interactions, ${ }^{[6]}$ and led to 1980 Nobel prizes for J. Cronin and V. Fitch. To date, in spite of a considerable number of experimental investigations, no violations of $C P T$ symmetry have ever been observed. ${ }^{[7]}$

\section{INCORPORATING CP VIOLATION INTO THE STANDARD MODEL}

Incorporating $C P$ violation $(C P V)$ into the Standard Model (SM), the current theory for the interactions of elementary particles, while preserving CPT proved not to be very easy. Wolfenstein proposed a mechanism that expanded the SM by adding a fifth, "superweak" force that produced a $C P$-violating off-diagonal contribution to the $2 \times 2$ neutral-kaon mass matrix and nothing else. ${ }^{[8]}$ However, the superweak explanation was ruled out by observations of direct $C P V$ decays of neutral kaons by the NA31 experiment at CERN ${ }^{[9]}$ and the KTeV experiment at Fermilab. ${ }^{[10]}$
To incorporate $C P V$ into the SM properly, one needs an amplitude that has a complex phase $\phi_{C P}$ that has opposite signs for particle and antiparticle processes. Since measureable processes are proportional to the absolute square of the amplitude, this $C P V$ phase is unmeasureable unless the $C P$-violating process interferes with another process that has a non-zero "strong," or "common" phase $\delta_{0}$, that has the same sign for particles and antiparticles. ${ }^{[1]}$ This is illustrated in Fig. 2a, where a hypothetical $C P$ violating process $X^{0} \rightarrow \pi^{+} \pi^{-}\left(\bar{X}^{0} \rightarrow \pi^{-} \pi^{+}\right)$has a complex amplitude $\mathcal{A}=|\mathcal{A}| \exp \left(i \phi_{C P}\right)\left(\overline{\mathcal{A}}=|\mathcal{A}| \exp \left(-i \phi_{C P}\right)\right)$. Differences in the decay rates can be measured if the $C P$-violating amplitude interferes with a $C P$-conserving amplitude for the same process $C=|C| \exp \left(i \delta_{0}\right)=\bar{C}$. In that case the $X^{0} \rightarrow \pi^{+} \pi^{-}$ and $\bar{X}^{0} \rightarrow \pi^{-} \pi^{+}$decay rates differ by a term proportional to $2|\mathcal{A}||C| \sin \delta_{0} \sin \phi_{C P}$; note that this interference term is zero (and $C P V$ remains unmeasureable), if $\delta_{0}=0$.

\section{The Kobayashi-Maskawa six-quark scheme for CP violation}

In 1973, Kobayashi and Maskawa (KM) showed that a non-trivial $C P$-violating phase could be introduced into the weak interaction quark-flavor mixing matrix, but only if there were at least three generations of quark doublets, i.e., at least six quark flavors (see Fig. 2b). ${ }^{[12]}$ This was remarkable because at that time, only three quark flavors had been established. In 1974, soon after the KM paper appeared, the fourth quark, the $c$-quark, was discovered at Brookhaven ${ }^{[13]}$ and SLAC $^{[14]}$ and the fifth quark, the $b$-quark, was found in 1977 by a Fermilab experiment. ${ }^{[15]}$ By that time everyone was convinced

\section{REFERENCES}

[6] J. H. Christenson, J. W. Cronin, V. L. Fitch and R. Turlay, Phys. Rev. Lett. 13, 138 (18964).

[7] T. Higuchi et al. (Belle Collaboration), Phys. Rev. D 85, 071105(R) (2012), and references cited therein.

[8] L. Wolfenstein, Phys. Rev. Lett. 13, 562 (1964).

[9] G. D. Barr el al. (NA31 Collaboration), Phys. Lett. B317, 233 (1993).

[10] A. Alavi-Harati et al. (KTeV Collaboration), Phys. Rev. Lett. 83, 22 (1999).

[11] T. Brown, S. Pakvasa and S. F. Tuan, Phys. Rev. Lett. 51, 1823 (1983).

[12] M. Kobayashi and T. Maskawa, Prog. Theor. Phys. 49, 652 (1973)

[13] J. J. Aubert et al., Phys. Rev. Lett. 33, 1404 (1974).

[14] J. E. Augustin et al. (Mark I Collaboration), Phys. Rev. Lett. 33, 1406 (1974).

[15] S. W. Herb et al., Phys. Rev. Lett. 39, 252 (1977) 


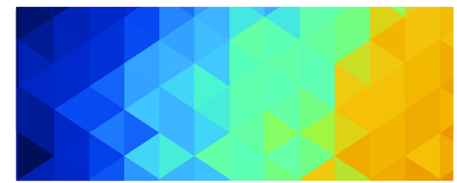

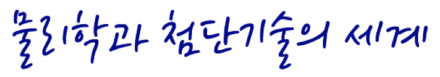

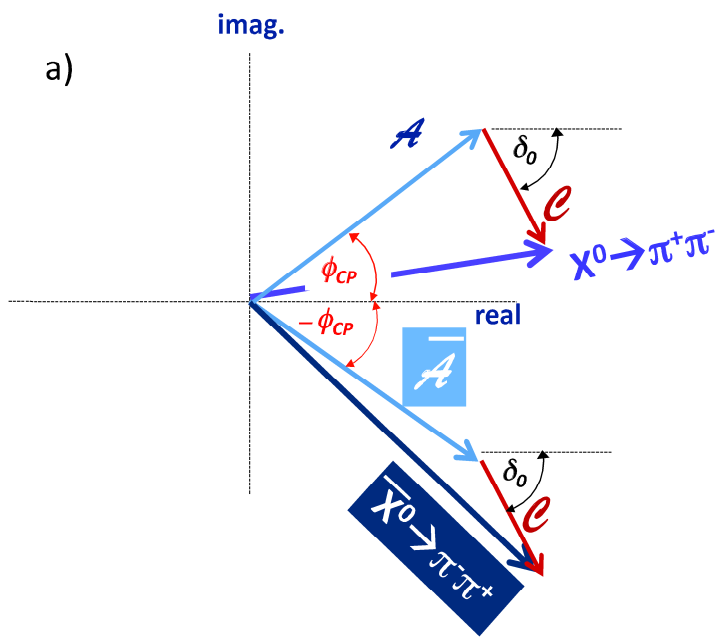

b)

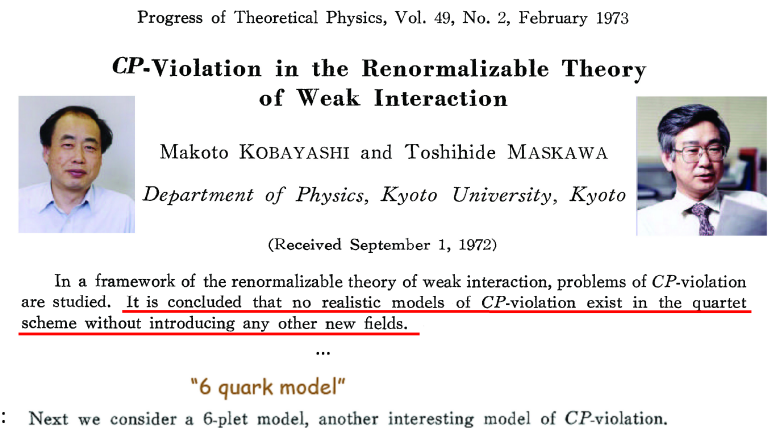

Page 12: Next we consider a 6-plet model, another interesting model of $C P$-violation.

$$
\begin{aligned}
\left(\begin{array}{l}
d^{\prime} \\
s^{\prime} \\
b^{\prime}
\end{array}\right)= & \left(\begin{array}{ccc}
c_{12} c_{13} & s_{12} c_{13} & s_{13} e^{-i \delta} \\
-s_{12} c_{23}-c_{12} s_{23} s_{13} e^{i \delta} & c_{12} c_{23}-s_{12} s_{23} s_{13} e^{i \delta} & s_{23} c_{13} \\
s_{12} s_{23}-c_{12} c_{23} s_{13} e^{i \delta} & -c_{12} s_{23}-s_{12} c_{23} s_{13} e^{i \delta} & c_{23} c_{13}
\end{array}\right)\left(\begin{array}{l}
d \\
s \\
b
\end{array}\right) \\
& \text { 3 Euler angles: } \theta_{1} \theta_{2} \& \theta_{3} \text {, plus } 1 \text { CP-violating phase: } \delta
\end{aligned}
$$

Fig. 2. a) The amplitude $\mathcal{A}$ represents a $C$ Pviolating contribution to a hypothetical process $X^{0} \rightarrow \pi^{+} \pi^{-}$. Differences in the $X^{0} \rightarrow \pi^{+} \pi^{-}$and $\bar{X}^{0} \rightarrow \pi^{-} \pi^{+}$ decay rates can only be observed if it interferes with a $C$ conserving process (amplitude $=C$ ) that has a non-zero common phase $\delta_{0}$. b) Excerpts from page 1 (above) and page 12 (below) of the classic Kobayashi-Maskawa paper. ${ }^{[12]}$

that the sixth quark, the top or $t$-quark, existed and it was only a matter of finding it. (It was found at Fermilab in 1995. ${ }^{[16,17]}$ ) Now, the commonly used form of the KM mixing matrix (now called the CKM matrix, where C designates Cabibbo, an originator of the quark-flavor-mixing idea $\left.{ }^{[18]}\right)$ is:

$$
\begin{aligned}
\left(\begin{array}{l}
d^{\prime} \\
s^{\prime} \\
b^{\prime}
\end{array}\right) & =\left(\begin{array}{lll}
V_{u d} & V_{u s} & V_{u b} \\
V_{c d} & V_{c s} & V_{c b} \\
V_{t d} & V_{t s} & V_{t b}
\end{array}\right)\left(\begin{array}{l}
d \\
s \\
b
\end{array}\right) \\
& =\left(\begin{array}{crc}
0.9743 & 0.2254 & 0.0036 e^{-i \phi_{3}} \\
-0.2252 & 0.9734 & 0.0414 \\
0.0089 e^{i \phi_{1}} & -0.0405 & 0.9991
\end{array}\right)\left(\begin{array}{l}
d \\
s \\
b
\end{array}\right),
\end{aligned}
$$

which rotates the mass eigenstates $(d, s, b)$ of down-like quarks of charge $-1 / 3|e|$ into their weak-interaction eigenstates $\left(d^{\prime}, s^{\prime}, b^{\prime}\right)$. Here $\left|V_{i j}\right|^{2}$ represents the probability that the $i^{\text {th }}$ up-like quarks of charge $+2 / 3|e|$ decays into the $j^{\text {th }}$ down-like quark. In this scheme, $V_{t d}$ and $V_{u b}$ are complex and have $C P$-violating phases $\phi_{1}$ and $\phi_{3}$, respectively, and the other seven matrix elements are real; the numerical values shown for reference on the right are the central values from the 2014 Particle Databook. ${ }^{[19]}$

\section{Testing the Kobayashi-Maskawa idea}

In a 1980 paper, Carter and Sanda pointed out that if the $b$-quark-related flavor-mixing parameters were such that the $B^{0}$ $\leftrightarrow \bar{B}^{0}$ particle-antiparticle mixing frequency was substantial and the $B$-meson lifetime was relatively long, large $C P V$ might be observable in neutral $B$ meson decays and provide con- clusive tests of the KM idea. ${ }^{[20]}$ However, that tests that Carter and Sanda proposed would require data samples that contained several hundreds of exclusive $B^{0}$ decays to $C P$ eigenstates, such as $B^{0} \rightarrow K_{S} J / \psi$, where $K_{S}$ is the "Short-lived" neutral $K$ meson and $J / \psi$ is the $1^{3} S_{1} c \bar{c}$ meson. In 1983, CLEO reported the world's first sample of exclusive $B$-meson decays shown in Fig. 3a, where there are only 18 events in the $B$-meson mass peak, divided equally between neutral and charged $B$-mesons and with a background that is estimated to be between 4 and 7 events. ${ }^{[21]}$ No exclusive decays to a $C P$ eigenstate were observed. Thus, in the early 1980's, when the state-of-the-art $e^{+} e^{-}$collider luminosity was $\sim 10^{31} \mathrm{~cm}^{-2} \mathrm{~s}^{-1}$, the possibility for checking the KM idea seemed hopeless, except for a few super-optimists who could foresee a more than a hundred-fold increase in luminosity to greater than $10^{33} \mathrm{~cm}^{-2} \mathrm{~s}^{-1}$ in less than two decades.

For the 1973 KM idea to be testable: six quark flavors had to exist in contrast to the three that were known at that time; a relatively long $B$-meson lifetime and a sizable $B^{0} \leftrightarrow \bar{B}^{0}$ mixing probability had to occur; and there had to be a thou-

\section{REFERENCES}

[16] S. Abachi et al. (D0 Collaboration), Phys. Rev. Lett. 74, 2422 (1995).

[17] F. Abe et al. (CDF Collaboration), Phys. Rev. Lett. 74, 2626 (1995).

[18] N. Cabibbo, Phys. Rev. Lett. 10, 531 (1963).

[19] K. A. Olive et al. (Particle Data Group), Chin. Phys. C 38 , 090001 (2014)

[20] A. B. Carter and A. I. Sanda, Phys. Rev. Lett. 45, 952 (1980).

[21] Phys. Rev. Lett. 50, 881 (1983). 

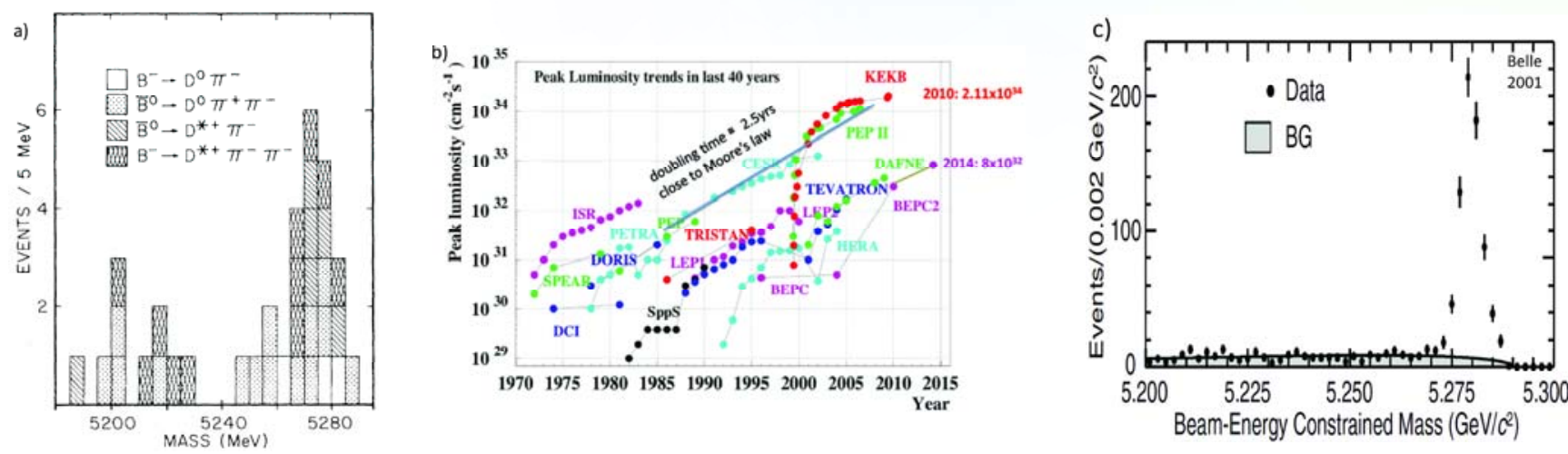

Fig. 3. a) (Figure 2 from ref. [21].) The first reported signal for exclusive $B$-mesons decays, found by CLEO in a $40 \mathrm{pb}^{-1}$ data sample recorded over a three-year time period. b) A "Livingston plot" for $e^{+} e^{-}$luminosities vs. year. c) (Figure 1 from ref. [22].) The $2001 B \rightarrow K_{S}(c \bar{C}), \xi_{f}=-1$ $C P$ eigenstate decay signal from a $29 \mathrm{fb}^{-1}$ Belle data sample, containing $\sim 700$ signal events, mostly $B \rightarrow K_{S} J / \psi$ decays, with a $92 \%$ signal purity.

sand-fold (or more) combined improvement in $e^{+} e^{-}$luminosity and detector performance. In 1983 , a long $\left(\tau_{B} \simeq 1.5 \mathrm{ps}\right)$ $B$-meson lifetime was measured at $\operatorname{SLAC}^{[23,24]}$ and, in 1987, a substantial signal for $B^{0} \leftrightarrow \bar{B}^{0}$ mixing was unexpectedly discovered by the ARGUS experiment at DESY. ${ }^{[25]}$ Taken together, these results indicated that the CKM mixing-angle values were favorable for experimental tests of the KM idea. In addition, the luminosity of $e^{+} e^{-}$colliders kept increasing in a Moore's-law-like fashion with a doubling time of about 2.5 years (see Fig. 3b). Detector performance also improved but at a less spectacular rate. In 2001, less than twenty years after the CLEO report of an 18 event exclusive $B$-meson decay signal with no $C P$ eigenstate modes, the Belle experiment's discovery paper on $C P V$ in the $B$-meson system was based primarily on the $\sim 700$ neutral $B$ mesons to $C P$ eigenstate decays with $C P$ eigenvalue $\xi_{f}=-1$ (mostly $B \rightarrow K_{S} J / \psi$ ) in the signal peak shown in Fig. 3c. ${ }^{[22]}$

\section{EXPERIMENTAL TECHNIQUE AND CHALLENGES}

In 1981, Bigi and Sanda ${ }^{[26]}$ suggested that $\phi_{1}$, the CPVphase of $V_{t d}$, could be measured by the interference between the two $B^{0}$-meson quark-line processes shown in Fig. 4a. Here the top diagram is the direct $B^{0}$-meson decay to a $C P$ eigenstate (chosen here as $K_{S} J / \psi$ for illustration). In the lower diagram, the $B^{0}$ first mixes into a $\bar{B}^{0}$ and the $\bar{B}^{0}$ decays to the same $C P$ eigenstate. The amplitude for the upper diagram is proportional to $V_{c b}$, which has no $C P V$ phase; that for the lower diagram is proportional to $V_{t b}^{2} V_{t d}^{* 2} V_{u b}$, where, in the KM formalism, only $V_{t d}^{*}$ has a $C P$ violating phase. Thus, the interference term between the two processes is proportional to $V_{t d}^{* 2}$, in other words, $\sin 2 \phi_{1}$.

The way this interference is measured is illustrated in Fig. 4b. An asymmetric energy $e^{+} e^{-}$collison produces a boosted $B^{0}$ and $\bar{B}^{0}$ in a "entangled" $J^{P C}=1^{--}$quantum state. After some time, one of the $B$ meson decays to a "flavor specific" final-state, i.e. a final state that allows one to distinguish (tag) whether the flavor of decaying $B$ meson is a $B^{0}$ or a $\bar{B}^{0}$. At that time, which is taken as $t=0$, the accompanying $B$ meson has to have the opposite flavor. Then this accompanying $B$ meson evolves with time, mixing as it goes along (either forward or backward in time!) into the opposite flavor with a frequency $\omega_{\text {mix }}$, and eventually decays at time $t$ into a CPeigenstate. What is measured, is the asymmetry $\mathcal{A}_{C P}$ as a function of $t$, where

$$
\mathcal{A}_{C P}=\frac{N_{\bar{B}^{0}}-N_{B^{0}}}{N_{\bar{B}^{0}}+N_{B^{0}}}=\xi_{f} \mathcal{E}(1-2 w) \sin 2 \phi_{1} \sin \omega_{\text {mix }} t .
$$

Here $N_{B^{0}}\left(N_{\bar{B}^{0}}\right)$ is the number of times the flavor-tagged $B$ is a $B^{0}\left(\bar{B}^{0}\right), \xi_{f}$ is the $C P$ eigenvalue of the state being studied (for $B \rightarrow K_{S} J / \psi, \xi_{f}=-1$ ), $\varepsilon$ is the efficiency for tagging the flavor of the accompanying $B$ meson, $w$ is the probability that the flavor-tagged $B$ meson is assigned the wrong flavor, $\omega_{\text {mix }}$ is the $B^{0} \leftrightarrow \bar{B}^{0}$ mixing frequency, which is well meas-

\section{REFERENCES}

[22] K. Abe et al. (Belle Collaboration), Phys. Rev. Lett. 87, 091802 (2001).

[23] E. Fernandez et al. (MAC Collaboration), Phys. Rev. Lett. 51, 1022 (1983).

[24] N. Lockyer et al. (Mark II Collaboration), Phys. Rev. Lett. 51, 1316 (1983).

[25] H. Albrecht et al. (ARGUS Collaboration), Phys. Lett. B 192, 245 (1987)

[26] I. I. Bigi and A. I. Sanda, Nucl. Phys. B 193, 85 (1981) 
a)
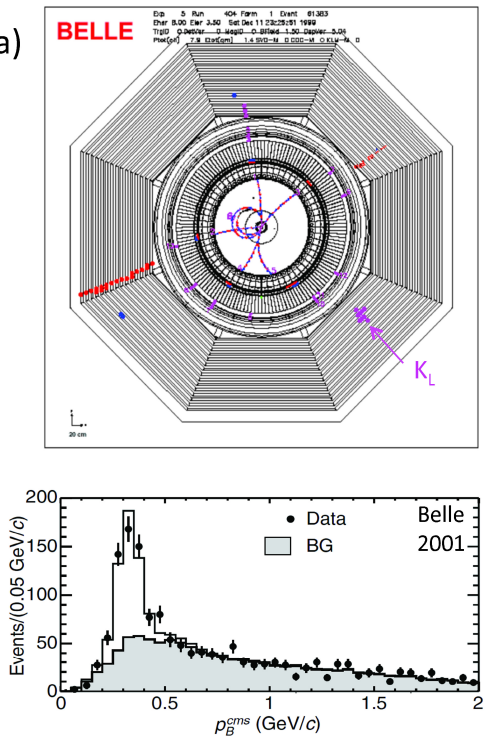

b)

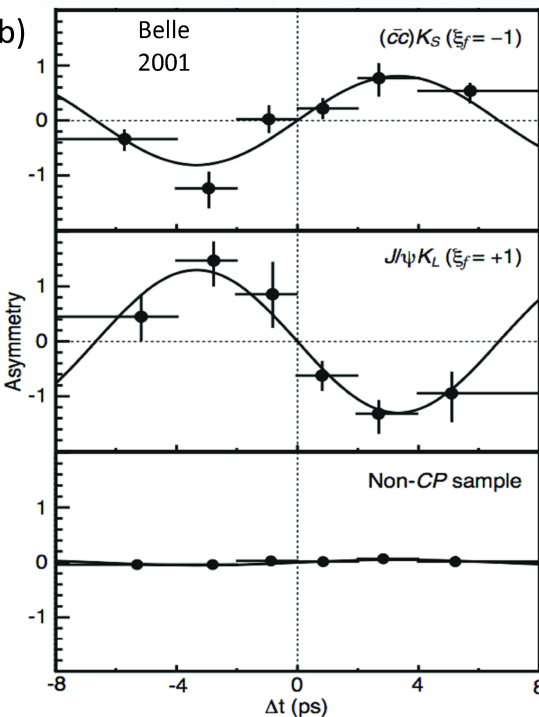

c)

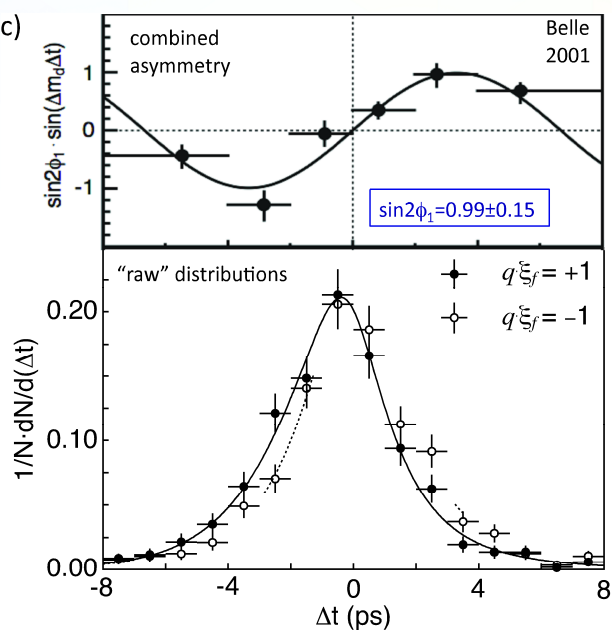

Fig. 5. a) (top) A computer display of $B \rightarrow K_{L} J / \psi$ events candidate in Belle, where the $J / \psi$ decays into a $\mu^{+} \mu^{-}$pair and the $K_{L}$ produces an energy cluster in the magnet's instrumented flux return. (bottom) The $p_{B}^{\text {cms }}$ distribution for candidate $K J / \psi$ events. b) The $t$-dependent $C P$ asymmetry for $\xi$, $=-1$ (top), $\xi_{f}=+1$ (center) and non- $C P$ eigenstate decays (bottom). c) The $t$ dependence of events organized according to $q \xi_{f}$ values, where $q=+1(-1)$ corresponds to a tagged $B^{0}\left(\bar{B}^{0}\right)$ (bottom). The combined $q \xi_{f}=-1$ minus $q \xi_{f}=+1$ asymmetries, together with the fit results (top).

pared with constraints from other measurements from completely different processes in Fig. 6a, where a region of common overlap, shown in yellow, is evident. ${ }^{[28]}$ Eventually BaBar and Belle accumulated huge amounts of additional data and significantly improved the precision on their $\phi_{1}$ measurements; their current average is $\sin 2 \phi_{1}=0.68 \pm 0.02 .{ }^{[19]}$ Since the largest possible value for $\left|\sin 2 \phi_{1}\right|$ is unity, the measured $\sin 2 \phi_{1}$ value qualifies the $C P V$ in $B$ meson decays as being "large," as first suggested by Carter and Sanda. Figure $6 \mathrm{~b}$ shows the currently allowed $\phi_{1}$ region together with more up-to-date constraints from measurements of the related quantities in the 2016 version of the same plot; ${ }^{[29]}$ the fact that all the bands in the figure overlap at the now much smaller common region (shown in yellow) demonstrates the consistency of the KM picture.

\section{Implications}

The Belle and BaBar results demonstrate that the Kobayashi Maskawa mechanism can account for all of the CPV that have been experimentally observed to date. However, it fails to account for the observed baryon asymmetry of the Universe by many orders of magnitude, which suggests that other $C P V$ mechanisms must exist in nature. One possibility is in the neutrino sector, where the $3 \times 3$ Pontecorvo-Maki-Nakagawa-Sakata (PMNS) neutrino-flavor-mixing matrix likely has KM-like CPV phases: one if the neutrinos are Dirac particles and two if they are Majorana-type. For the Majorana case, neutrino-induced $C P V$ processes in the early Universe can, in principle, be large enough to account for the baryon asymmetry of the Universe. ${ }^{[31]}$ This possibility, called "Leptogenesis," is being investigated by experiments in Japan ${ }^{[32]}$ and the U.S. ${ }^{[33]}$ Other possibilities are modifications to the SM that include particles that are too massive to be accessible with current day accelerators, but could contribute higher-order quantum-loop effects in rare decays of $B$ mesons that could be etablished by precision measurements by the LHCb experiment at CERN ${ }^{[34]}$ and/or the BelleII experiment that is in its final stages of preparation at KEK in Japan. ${ }^{[35]}$

\section{REFERENCES}

[27] B. Aubert et al. (BaBar Collaboration), Phys. Rev. Lett. 87, 091801 (2001)

[28] J. Charles et al. (CKM-Fitter Group), Eur. Phys. J. C 41, 1 (2005)

[29] Ed. A. J. Bevan, B. Golob, Th. Mannel, S. Prell and B. D. Yabsley, Eur. Phys. J. C74, 3026 (2014).

[30] L. Wolfenstein, Phys. Rev. Lett. 51, 1945 (1983).

[31] M. Fukugita and T. Yanagida, Phys. Lett. B 174, 45 (1986).

[32] K. Abe et al. (T2K Collaboration), Nucl. Instr. Meth. A 659, 106 (2011).

[33] D. S. Ayres et al. (NOvA Collaboration), The NOvA Technical Design Report, FERMILAB-DESIGN-2007-1.

[34] A. Augusto Alves et al. (LHCb Collaboration), JINST 3, S08005 (2008).

[35] T. Aushev et al. (Bellell Collaboration), arXiv:1002.5012 [hepex] (2010) 


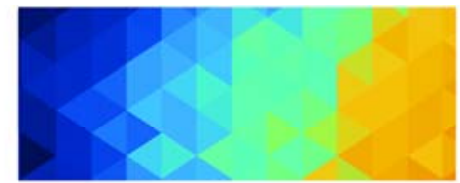

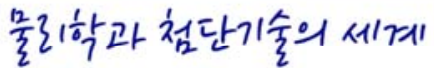
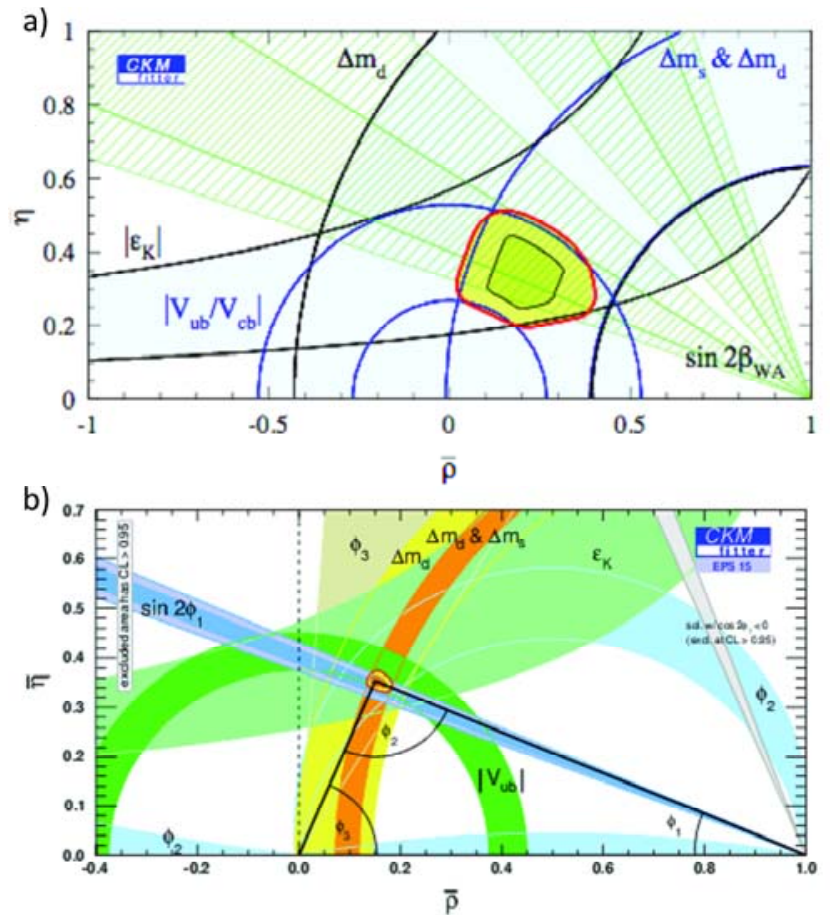

Fig. 6. a) The unitarity triangle plot from the CKM-fitter group with the average of the $2001 \mathrm{BaBar}$ and Belle $\sin 2 \phi_{1}$ results (labeled as $\left.\sin 2 \beta_{W A}\right)$. Here $\bar{\rho}$ and $\bar{\eta}$ are parameters introduced by Wolfenstein ${ }^{[30]}$ that characterize the real and imaginary parts, respectively, of $V_{u b}=$ $0.0094(\rho-i \eta)$ and $V_{t d}=0.0094(1-\rho-i \eta)$. The band labeled $\left|\epsilon_{K}\right|$ is the constraint from measurements of CPV in $K_{L}$ decay, those labeled $\Delta m_{d}$ and $\Delta m_{s}$ are constraints from $B^{0} \leftrightarrow \bar{B}^{0}$ and $B_{s}{ }^{0} \leftrightarrow \bar{B}_{s}{ }^{0}$ mixing frequency measurements, and the annular band centered at zero are constraints from $\left|V_{u b}\right|$ measurements. b) The 2015 version of the CKM-fitter group's unitary triangle plot.

\section{AFTERWORD}

What happened to the principal players? Andrei Sakharov won the 1975 Nobel Peace Prize, but was not allowed to leave the USSR to receive it. His human rights activities led to his confinement to the closed city of Gorki (now Nizhny Novgorod) from 1980 to 1986 . He died in Moscow in 1989. Susumu Okubo won the 1976 Nishina Memorial Prize, the 2005 Sakurai Prize and the 2006 Wigner Medal. He died in 2015. In December 2008, Makoto Kobayashi and Toshihide Maskawa met the King of Sweden (see Fig. 7a) and are currently big celebrities in Japan. Anthony Ichiro Sanda (Fig. 7b left) is retired and living in Japan, where he is heavily engaged in church-related activities. Ikaros Bigi (Fig. 7b right) continues to teach and do research at Notre Dame University in the U.S. Ashton Carter (Fig. 7c), who was Sanda's post-doc in 1980-1981, switched from theoretical physics into national security-related matters shortly thereafter and, most recently, a)

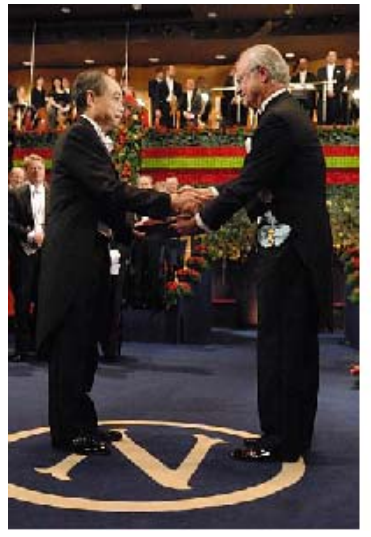

b)
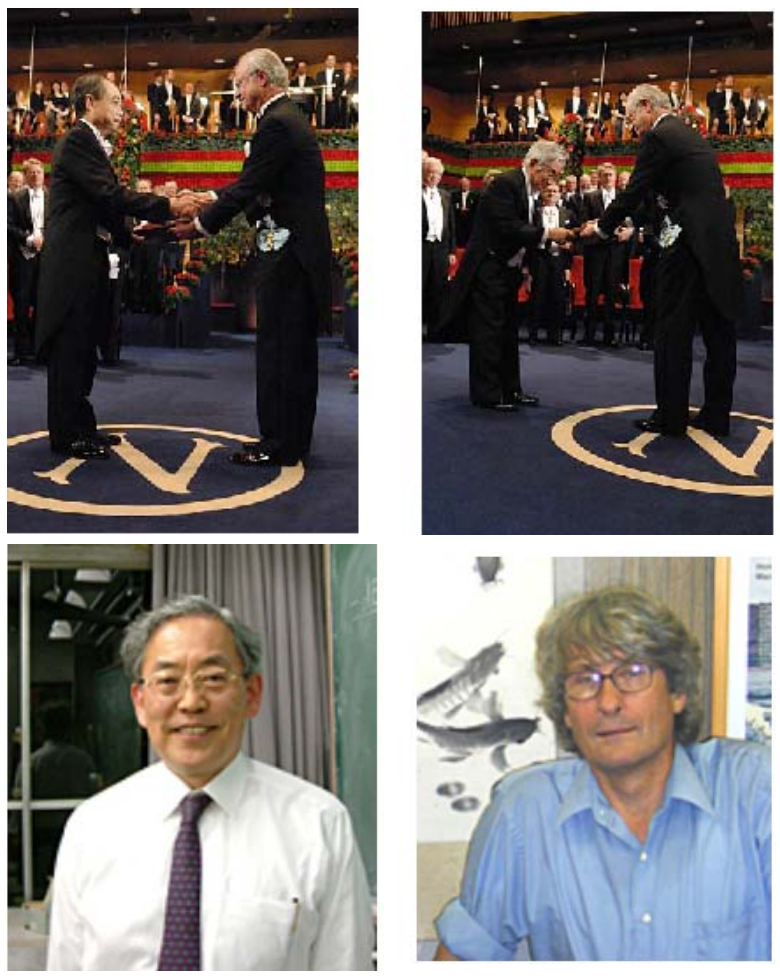

c)

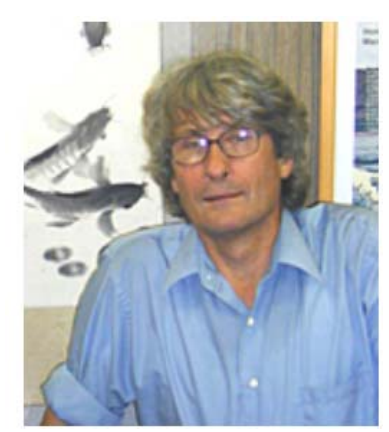

(J) 중앙일보 2015.04.2015

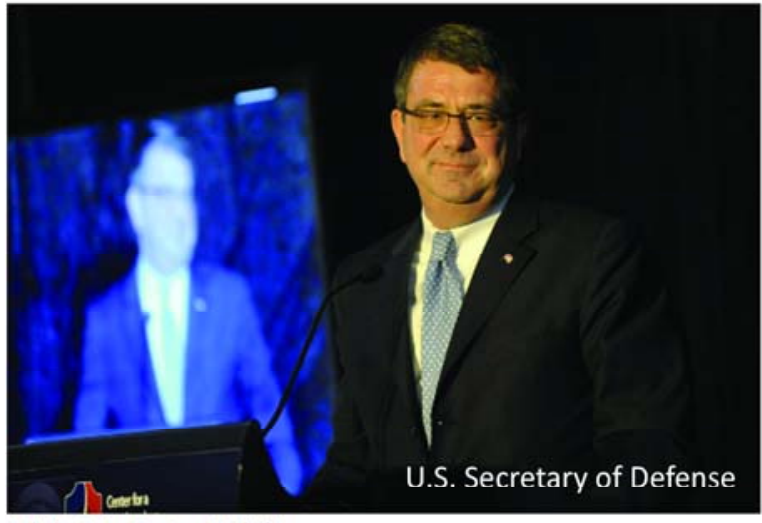

(사진=Ash Carter 플리커)

$=$ 예, 미 국방장관의 방한.

Fig. 7. a) Kobayashi (left) and Maskawa (right) receiving 2008 Nobel physics prizes from King Carl XVI Gustav of Sweden. b) Sanda (left) and Bigi (right) were co-recipients of the 2004 U.S. APS' prestigious J. J. Sakurai prize for theoretical physics. c) Carter was appointed U.S. Secretary of Defense in February 2014.

has been working for the U.S. Department of Defense.

\section{Acknowledgments}

This work was supported by the Institute for Basic Science (Korea) under project code IBS-R016-D1. 